\title{
Comparative Performance of PLZT and PVDF Pyroelectric Sensors Used to the Thermal Characterization of Liquid Samples
}

\author{
Gemima Lara Hernandez, ${ }^{1,2}$ Alfredo Cruz-Orea, \\ Ernesto Suaste Gomez, ${ }^{1}$ and Jose Jesus Agustin Flores Cuautle ${ }^{1,3}$ \\ ${ }^{1}$ Electrical Engineering Department, CINVESTAV-IPN, Avenue IPN 2508, San Pedro Zacatenco, Gustavo A. Madero, \\ 07360 Mexico City, DF, Mexico \\ ${ }^{2}$ Physics Department, CINVESTAV-IPN, Avenue IPN 2508, San Pedro Zacatenco, Gustavo A. Madero, 07360 Mexico City, DF, Mexico \\ ${ }^{3}$ Laboratory for Acoustics and Thermal Physics, Department of Physics and Astronomy, KU LEUVEN, Celestijnenlaan 200D, 3001 \\ Leuven, Belgium
}

Correspondence should be addressed to Jose Jesus Agustin Flores Cuautle; jjflores@cinvestav.mx

Received 31 May 2013; Accepted 21 September 2013

Academic Editor: Said Abdallah

Copyright (C) 2013 Gemima Lara Hernandez et al. This is an open access article distributed under the Creative Commons Attribution License, which permits unrestricted use, distribution, and reproduction in any medium, provided the original work is properly cited.

Among the photothermal methods, the photopyroelectric (PPE) technique is a suitable method to determine thermal properties of different kinds of samples ranging from solids to liquids and gases. Polyvinylidene difluoride (PVDF) is one of the most frequently used pyroelectric sensors in PPE technique but has the disadvantage that it can be easily deformed by the sample weight. This deformation could add a piezoelectric effect to the thermal parameters assessment; also PVDF has a narrow temperature operation range when compared with ceramic pyroelectric sensors. In order to minimize possible piezoelectric effects due to sensor deformation, a ceramic of lanthanum modified lead zirconate (PLZT) was used as pyroelectric sensor in the PPE technique. Then, thermal diffusivity of some liquid samples was measured, by using the PPE configuration that denominated the thermal wave resonator cavity (TWRC), with a PLZT ceramic as pyroelectric detector. The performance obtained with the proposed ceramic in the TWRC configuration was compared with that obtained with PVDF by using the same configuration.

\section{Introduction}

In recent years, the photothermal (PT) methods have developed useful experimental configurations in order to evaluate the thermal and optical properties of materials [1]. PT methods can provide direct optical absorption spectra, also the characterization of thermal properties, and analysis of nonradiative relaxation processes. These methods also have been used to determine thermal parameters of solid, liquid, and gaseous samples [1].

Among the PT methods used to examine thermal properties of liquids stands the thermal lens (TL) spectrometry. This PT technique has been used to study nearly transparent liquids, with the disadvantage that light is scattered when solid particles are suspended into the liquid [2]; in order to avoid light scattering, an indirect sample heating can be used by using another PT method as the PPE technique. In the present study is described the thermal wave resonator cavity (TWRC) method, based on a PPE detection, using a pyroelectric ceramic as a sensor in order to improve the pyroelectric detection of thermal waves.

The PPE technique is used to evaluate thermal properties in different materials, especially in liquids. One of the most widely used PPE configurations is the direct (or back) detection configuration. In the back configuration, the thermal diffusivity of the sample $\left(\alpha_{s}\right)$ can be obtained. In the case of liquid samples which are enclosed in a cavity formed by the pyroelectric (PE) detector and a metal light absorber. Thermal waves generated in the metal surface due to the absorption of a laser beam are detected with a PE sensor. PE 


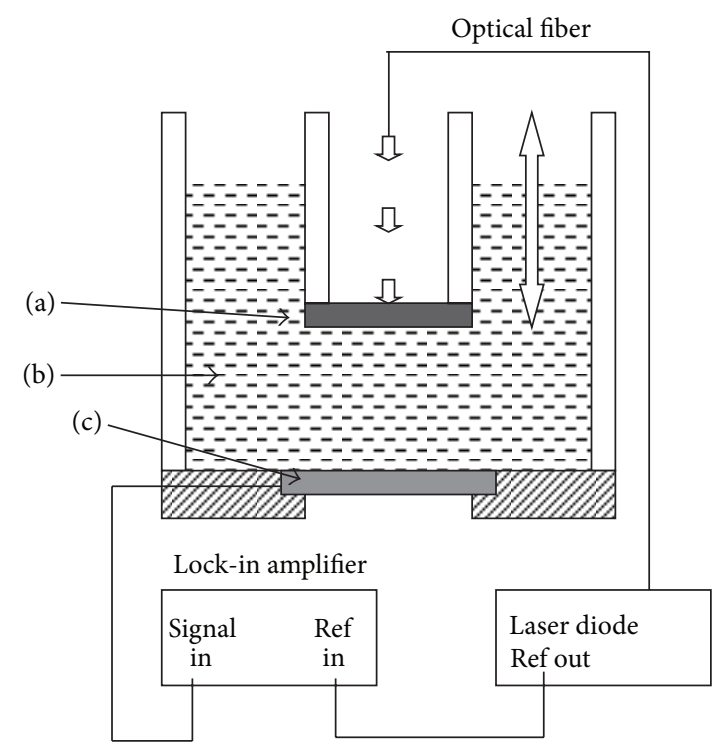

FIGURE 1: Schematic representation of the experimental setup of TWRC, (a) copper foil, (b) liquid sample, and (c) PE detector.

signal amplitude is proportional to the temperature variation, and it is related to $\alpha$ in a straightforward way; this technique is known as thermal wave resonator cavity [3-5].

TWRC is an experimental setup used to obtain thermal diffusivity of liquids with high precision $[3,6]$; in this technique cavity length (formed by PE sensor and metal thermal wave generator) is scanned with fixed light modulation frequency. Scanning cavity length instead of modulation frequency has the advantage that signal to noise ratio is improved, as well as disposing with the requirement of instrumental transfer function normalization.

TWRC is based on a pyroelectric sensor, usually polyvinylidene difluoride (PVDF), which is an excellent detector but has the disadvantage that it can be easily deformed by the sample weight [7]. This deformation could add a piezoelectric effect in the calculation and then the theoretical modeling would become more complicated $[8,9]$. Usually, metal baking is used as a way to avoid piezoelectric effect with the consequence that PE signal amplitude diminishes [10]. A pyroelectric ceramic was proposed as PE sensor in order to avoid a possible piezoelectric effect in the PPE measurements.

Lanthanum modified lead zirconate (PLZT) is a ferroelectric ceramic which possesses high pyroelectric coefficient and a Curie temperature $\left(T_{c}\right)$ of about $180^{\circ} \mathrm{C}[11,12]$; therefore, in the present study, PLZT was used as pyroelectric detector in TWRC configuration. Then, the thermal diffusivity $(\alpha)$ of distilled water, olive oil, glycerol, and ethylene glycol was measured by using this PLZT ceramic, and these values were compared with the values previously measured with PVDF as PE detector.

\section{Experimental}

2.1. PLZT Ceramic Preparation. The oxide-mixing technique was used to create the ferroelectric ceramic of
$\mathrm{Pb}_{1-x} \mathrm{La}_{x}\left(\mathrm{Zr}_{1-y} \mathrm{Ti}_{y}\right)_{1-x / 4} \mathrm{O}_{3}$ with $x=0.09$ and $y=0.35$ (PLZT), which is usually denoted as $(9 / 65 / 35)[14,15]$.

The raw powders were mixed with an electronic mill (Pulverisette 2, Fritsch) during 20 minutes; some polyvinyl alcohol drops were added at a proportion of $0.075 \mathrm{~mL}$ per each gram of mixture. Then, the resulting powder was pressed, with a load of $3500 \mathrm{Kg} / \mathrm{cm}^{2}$, in order to obtain discs of $10 \mathrm{~mm}$ diameter and $2 \mathrm{~mm}$ thickness. These discs were sintered in air with a heating rate of $5^{\circ} \mathrm{C} / \mathrm{min}$ until they reached $600^{\circ} \mathrm{C}$, and then the heating rate was increased to $10^{\circ} \mathrm{C} / \mathrm{min}$ until they reached $1200^{\circ} \mathrm{C}$ during one hour into a platinum crucible. Once the ceramics were sintered, one of them was polished until reaching a final thickness of $756 \mu \mathrm{m}$.

Gold electrodes, with $10 \mathrm{~nm}$ thickness, were evaporated in the polished ceramic and afterwards this ceramic was poled under a DC electric field of $3 \mathrm{kV} / \mathrm{mm}$ by one hour in silicone oil bath. The resulting PLZT ceramic was used as pyroelectric sensor in the TWRC experimental setup in order to obtain the thermal diffusivity of well-know liquids as distilled water, olive oil, glycerol, and ethylene glycol.

2.2. Experimental Setup. The experimental setup used to obtain the sample thermal diffusivity $\left(\alpha_{s}\right)$ is shown in Figure 1.

This setup consisted of a chamber of variable length containing the liquid sample (b); cavity is formed by a circular $\mathrm{Cu}$ foil $100 \mu \mathrm{m}$ thick (a) and PLZT pyroelectric temperature sensor (c). A laser diode beam, modulated by the internal oscillator of a lock-in amplifier, impinges on the black painted inner surface of the metallic foil, which acts as light absorber.

As the intensity modulated light strikes on the metallic foil, its temperature fluctuates periodically at the same modulation frequency of the incident beam $(f)$; therefore, thermal waves travel to the liquid-filled chamber. The temperature oscillations at $x=l$ can be measured with the PE sensor as 


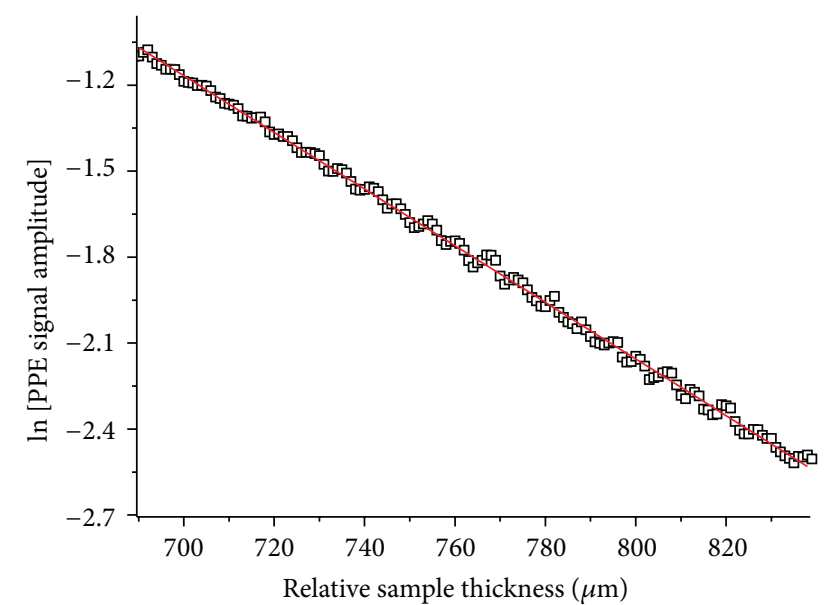

Figure 2: PPE signal amplitude as a function of the cavity length, having water as a sample and PVDF as PE detector; the solid line represents the best fitting of (2) to the experimental data.

a function of the liquid layer thickness. The PPE signal was fed into the lock-in amplifier, where its amplitude and phase are measured and processed by a personal computer in order to obtain the PPE signal as a function of sample thickness $\left(l_{s}\right)$. For a thermally thin copper foil $\left(a_{\mathrm{Cu}} l_{\mathrm{Cu}} \ll 1\right)$, thermally thick sample $\left(a_{s} l_{s} \gg 1\right)$, and thermally thick PE detector $\left(a_{p} l_{p} \gg 1\right)$ (where $a_{j}=\sqrt{\left(\pi f / \alpha_{j}\right)}$ and $l_{j}$ are the thermal diffusion coefficient and thickness of the $j$ th element in the PE cell), the output voltage can be expressed as [16]:

$$
V=A\left(\frac{\eta_{s} a_{p}}{\kappa_{p}\left(1+b_{s p}\right) \omega_{0}}\right) \exp \left[-a_{s} l_{s}\right] \exp \left\{-i\left[\frac{\pi}{2}+a_{s} l_{s}\right]\right\}
$$

where $A$ is an instrumental factor, $\eta_{s}$ is the nonradiative conversion efficiency for the absorbing solid, $\kappa_{p}$ is the thermal conductivity of the pyroelectric sensor, $b_{s p}=e_{s} / e_{p}$, with $e_{j}$ as the thermal effusivity of the $j$ th element in the PE cell, and $\omega_{0}$ is the angular frequency of the laser beam. When the experiment is running at fixed frequency of the modulated light beam, $A$ is constant as well as all the terms before the first exponential in (1), and all those terms are joined in a constant $(B)$; therefore, the output voltage can be reduced as

$$
V=B \exp \left[-a_{s} l_{s}\right] \exp \left\{-i\left[\frac{\pi}{2}+a_{s} l_{s}\right]\right\} .
$$

Then it is possible to obtain the sample thermal diffusivity $\left(\alpha_{s}\right)$ in a simple way by means of getting the slope of the logarithm of the PPE signal amplitude as function of $l_{s}$, in the same way the sample thermal diffusivity could be obtained by getting slope of the PPE signal phase also as a function of $l_{s}$.

The light modulation frequency $(f)$ used in the present study was chosen at $1.28 \mathrm{~Hz}$, in order to guarantee that sample and pyroelectric sensor were thermally thick; the light source was a diode laser beam, with $40 \mathrm{~mW}$ power and wavelength at $785 \mathrm{~nm}$. In the present study, the thermal diffusivities of different liquid samples were obtained by using the TWRC

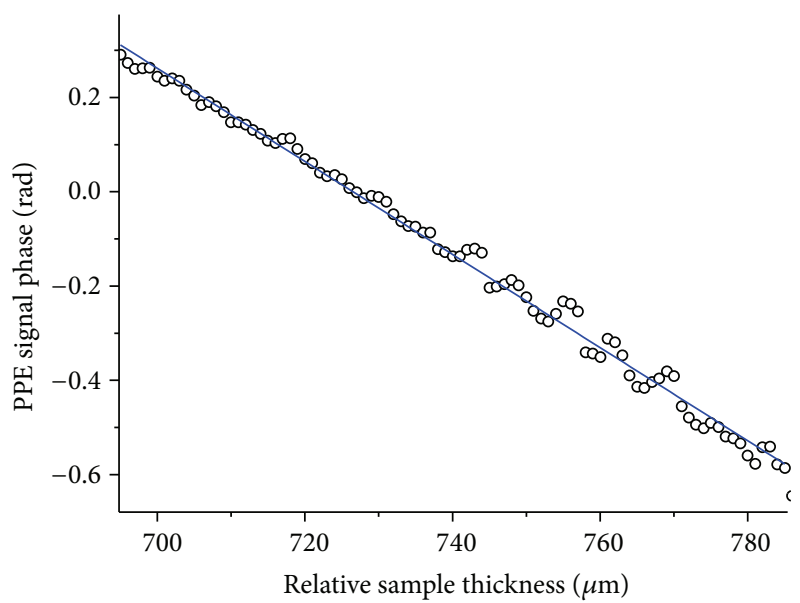

FIGURE 3: PPE signal phase as a function of the cavity length, having water as a sample and PVDF as PE detector; the solid line represents the best fitting of (2) to the experimental data.

with two different PE sensors (PLZT and PVDF) in order to compare their performance. For each PE sensor, the thermal diffusivity of the samples was obtained for both the PPE signal amplitude and phase data. The results obtained from each PE sensor were compared. All measurements were performed at $22^{\circ} \mathrm{C}$.

2.3. Samples. Samples used in this work were obtained as follows: edible oil (olive oil) was acquired from local food market and distillated water, glycerol, and ethylene glycol from a chemical supplier.

\section{Experimental Results and Discussion}

Figures 2 and 3 show the behavior of the PPE signal amplitude and phase as a function of the relative cavity length, respectively, using PDVF as PE detector in the case of distilled water. The solid line in Figure 2 represents the best fit of the natural logarithm of amplitude of (2) to the experimental natural logarithm of PPE signal amplitude; also the solid line in Figure 3 corresponds to the best fit of phase of (2) to the experimental PPE signal phase.

Figures 4 and 5 show the PPE signal amplitude and phase, respectively, as a function of the relative cavity length, by using now a PLZT ceramic as the PE detector also for distilled water; the solid line in Figures 4 and 5 represents the best fit of the amplitude and phase of (2) to the experimental PPE signal amplitude and phase data, respectively.

As it can be seen, from Figures 2 and 4, the range of the cavity length in which the theoretical model (amplitude of (2)) fits with the experimental amplitude data, is increased at least $400 \mu \mathrm{m}$ when PLZT detector is used instead of PVDF. This increase in the relative cavity length range results in a decrease of the error percentage.

In the case of the experimental phase data, the range of relative cavity length, in which the phase of (2) fits with the experimental data, is increased at least $500 \mu \mathrm{m}$, when PLZT detector is used instead of the PVDF detector (see 
TABLE 1: Thermal diffusivities of analyzed liquids.

\begin{tabular}{lccccc}
\hline \multirow{2}{*}{ Sample } & \multicolumn{2}{c}{ PLZT } & \multicolumn{2}{c}{ PVDF } & The literature \\
& $\alpha_{\text {Amp }}\left(\times 10^{-8} \mathrm{~m}^{2} / \mathrm{s}\right)$ & $\alpha_{\text {Phase }}\left(\times 10^{-8} \mathrm{~m}^{2} / \mathrm{s}\right)$ & $\alpha_{\text {Amp }}\left(\times 10^{-8} \mathrm{~m}^{2} / \mathrm{s}\right)$ & $\alpha_{\text {Phase }}\left(\times 10^{-8} \mathrm{~m}^{2} / \mathrm{s}\right)$ & $\alpha_{S}\left(\times 10^{-8} \mathrm{~m}^{2} / \mathrm{s}\right)$ \\
\hline Water & $14.23 \pm 0.049$ & $14.141 \pm 0.057$ & $14.13 \pm 0.13$ & $14.15 \pm 0.12$ & $9.16[13]$ \\
Glycerol & $9.19 \pm 0.050$ & $9.20 \pm 0.033$ & $9.22 \pm 0.14$ & $9.22 \pm 0.26$ & $9.13]$ \\
Ethylene glycol & $9.36 \pm 0.062$ & $9.36 \pm 0.030$ & $9.17 \pm 0.28$ & 9.11 & $8.18 \pm 3]$ \\
Olive oil & $8.91 \pm 0.043$ & $8.76 \pm 0.025$ & $8.76 \pm 0.053$ & 8.071 & $8.81[3]$ \\
\hline
\end{tabular}

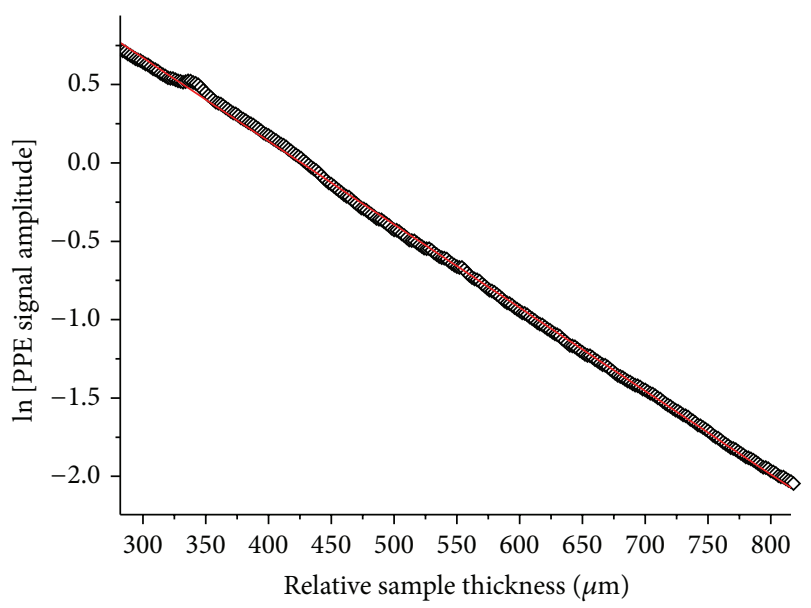

Figure 4: PPE signal amplitude as a function of the cavity length, having water as a sample and PLZT ceramic as PE detector; the solid line represents the best fitting of (2) to the experimental data.

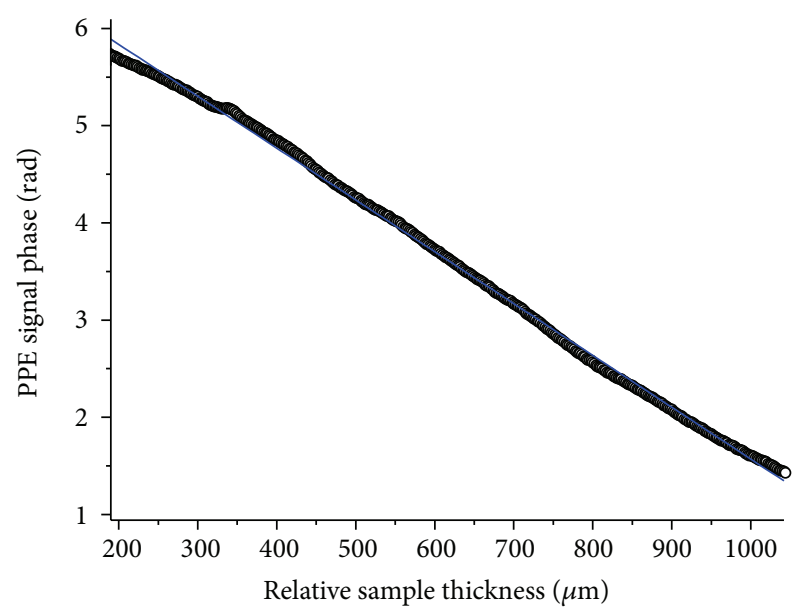

FIGURE 5: PPE signal phase as a function of the cavity length, having water as a sample and PLZT ceramic as PE detector; the solid line represents the best fitting of (2) to the experimental data.

Figures 3 and 5). In order to corroborate the enhancement of the measurements in TWRC configuration, when PLZT sensor is used instead of the PVDF sensor, the thermal diffusivities of other liquid samples (glycerol, ethylene glycol, and olive oil) were obtained by using the same procedure described prevoiusly and the results are shown in Table 1 where the obtained thermal diffusivities are compared, using PLZT and PVDF as PE detectors; it can be seen that the $\alpha_{s}$ values obtained with PLZT are near to the $\alpha_{s}$ values obtained with PVDF; however, an increment in the precision can be observed as well as a reduction of the errors.

It is common that, in PPE experimental setups, where a PVDF sensor is used, it is necessary to use a preamplifier before the PPE signal is sent to the lock-in amplifier $[3,8]$, while, in the proposed experimental setup with PLZT as sensor, this device can be omitted due to the high PPE signal provided by the PLZT detector. Also several authors have used $\mathrm{LiTaO}_{3}$ or $\mathrm{LiNbO}_{3}$ PE ceramics instead of PVDF foils due to their rigidity and higher Curie point $[17,18]$. In the case of $\mathrm{LiTaO}_{3}$ some authors have shown relative thickness scans for liquid samples ranged around $200 \mu \mathrm{m}$ [18]; in this range the PPE signal phase has a linear behavior as a function of $l_{s}$ according to (2) of the present paper, and in our case the linear behavior of the PPE signal phase, as a function of relative $l_{s}$, ranged around $900 \mu \mathrm{m}$, which allows a larger range of fitting of the theoretical equation (2) to the experimental data and, as a consequence, the error of this fitting would tend to a lower error. Also in the present study the ln (PPE signal amplitude) has a linear behavior, as a function of relative $l_{s}$, in a range that achieves around $600 \mu \mathrm{m}$; then also the PPE signal amplitude can be used to obtain the sample thermal diffusivity with a low error.

\section{Conclusions}

PLZT hardness prevents the deformations that in thin PVDF sheets occur due to the sample weight; these deformations could add a piezoelectric effect in the measurement. In this work, a PLZT was used as PE detector in order to obtain the thermal diffusivities of different liquid samples as distilled water, olive oil, glycerol, and ethylene glycol by the TWRC method. The obtained thermal diffusivities values are in agreement with those previously reported in the literature, which means that PLZT is a suitable candidate to be used as a pyroelectric detector in this technique. Besides this, the PLZT pyroelectric signal can be obtained in a wide frequency range, which means that the ceramic could be used to obtain thermal parameters of different samples.

The thermal diffusivity, calculated from the slope of the natural logarithm of PPE signal amplitude or from the PPE signal phase, as a function of the relative cavity length, theoretically most be the same according to (2); thus, a good indicator of the accuracy in the measurements is the ratio of 
obtained thermal diffusivities from amplitude and phase; for our measurements this ratio was near to 1 , and in the worst case was 0.98 , which indicates a very good approximation to the theoretical equation (2).

Another advantage of the ceramic PLZT over PVDF, for future studies, is the highest temperature range of operation, $80^{\circ} \mathrm{C}$ for PVDF and $180^{\circ} \mathrm{C}$ for PLZT, which allows obtaining thermal parameters at higher temperatures. In the present study, all measurements were made at room temperature, that is, far from the Curie temperature for the ceramic operation. Even when the PLZT detector is compared with other PE ceramics, it is possible to have higher signal to noise ratio for large thickness scans. Also, it is expected that PLZT will be a suitable sensor to obtain the thermal diffusivity as a function of the temperature in a wide temperature range for different samples.

\section{Acknowledgments}

This work was supported in part by the Mexican Agency CONACYT through the projects 151894 and 103632; J. J. A. Flores is also grateful to CONACYT for his postdoctoral grant. All authors are thankful to the following persons: Ing. E. Ayala, Ing. M. Guerrero, and Ing. A. B. Soto, of CINVESTAV-IPN, for their technical assistance.

\section{References}

[1] J. Caerels, C. Glorieux, and J. Thoen, "Absolute values of specific heat capacity and thermal conductivity of liquids from different modes of operation of a simple photopyroelectric setup," Review of Scientific Instruments, vol. 69, no. 6, pp. 2452-2458, 1998.

[2] J. Jiménez-Pérez, A. Cruz-Orea, J. Sánchez-Ramírez, F. Sánchez-Sinencio, L. Martínez-Pérez, and G. López Muñoz, "Thermal characterization of nanofluids with different solvents," International Journal of Thermophysics, vol. 30, no. 4, pp. 1227-1233, 2009.

[3] J. A. Balderas-López, A. Mandelis, and J. A. Garcia, "Thermalwave resonator cavity design and measurements of the thermal diffusivity of liquids," Review of Scientific Instruments, vol. 71, no. 7, pp. 2933-2937, 2000.

[4] A. Mandelis and M. M. Zver, "Theory of photopyroelectric spectroscopy of solids," Journal of Applied Physics, vol. 57, no. 9, pp. 4421-4430, 1985.

[5] M. Chirtoc and G. Mihilescu, "Theory of the photopyroelectric method for investigation of optical and thermal materials properties," Physical Review B, vol. 40, no. 14, pp. 9606-9617, 1989.

[6] B. B. Tepepa, E. Marín, E. S. Martín-Martínez, and A. C. Orea, "Thermal wave resonator cavity applied to the study of the thermal diffusivity of coffee infusions," International Journal of Thermophysics, vol. 30, no. 5, pp. 1591-1597, 2009.

[7] J. A. Balderas-Lopez, A. Mandelis, and J. A. Garcia, "Measurements of the thermal diffusivity of liquids with a thermal-wave resonator cavity," Analytical Sciences, vol. 17, pp. S519-S522, 2001.

[8] Piezo Film Sensors Technical Manual, Measurement Spcialities, Norristown, Pa, USA, 1999.
[9] W. Jackson and N. M. Amer, "Piezoelectric photoacoustic detection: theory and experiment," Journal of Applied Physics, vol. 51, no. 6, pp. 3343-3353, 1980.

[10] J. A. Balderas-López and A. Mandelis, "Self-consistent photothermal techniques: application for measuring thermal diffusivity in vegetable oils," Review of Scientific Instruments, vol. 74, no. 1, pp. 700-702, 2003.

[11] A. Peláiz Barranco, F. Calderón Piñar, and O. Pérez Martínez, "PLZT ferroelectric ceramics on the morphotropic boundary phase. Study as possible pyroelectric sensors," Physica Status Solidi A, vol. 186, pp. 479-485, 2001.

[12] J. D. Zook and S. T. Liu, "Use of effective field theory to predict relationships among ferroelectric parameters," Ferroelectrics, vol. 11, no. 1, pp. 371-376, 1976.

[13] G. Lara-Hernández, E. Suaste-Gómez, A. Cruz-Orea et al., "Thermal characterization of edible oils by using photopyroelectric technique," International Journal of Thermophysics, vol. 34, no. 5, pp. 962-971, 2013.

[14] I. A. Santos, A. L. Zanin, M. H. Lente et al., "Cerâmicas ferroelétricas transparentes de PLZT e PLMN-PT: processamento e propriedades," Cerâmica, vol. 49, no. 310, pp. 92-98, 2003.

[15] A. J. Moulson and J. M. Herbert, Electroceramics, John Wiley \& Sons, Chichester, UK, 2003.

[16] A. Mandelis and M. M. Zver, "Theory of photopyroelectric spectroscopy of solids," Journal of Applied Physics, vol. 57, no. 9, pp. 4421-4430, 1985.

[17] D. Dadarlat, "Photopyroelectric calorimetry of liquids. Recent development and applications," Laser Physics, vol. 19, no. 6, pp. 1330-1339, 2009.

[18] S. Delenclos, D. Dadarlat, N. Houriez, S. Longuemart, C. Kolinsky, and A. Hadj Sahraoui, "On the accurate determination of thermal diffusivity of liquids by using the photopyroelectric thickness scanning method," Review of Scientific Instruments, vol. 78, no. 2, Article ID 024902, 2007. 

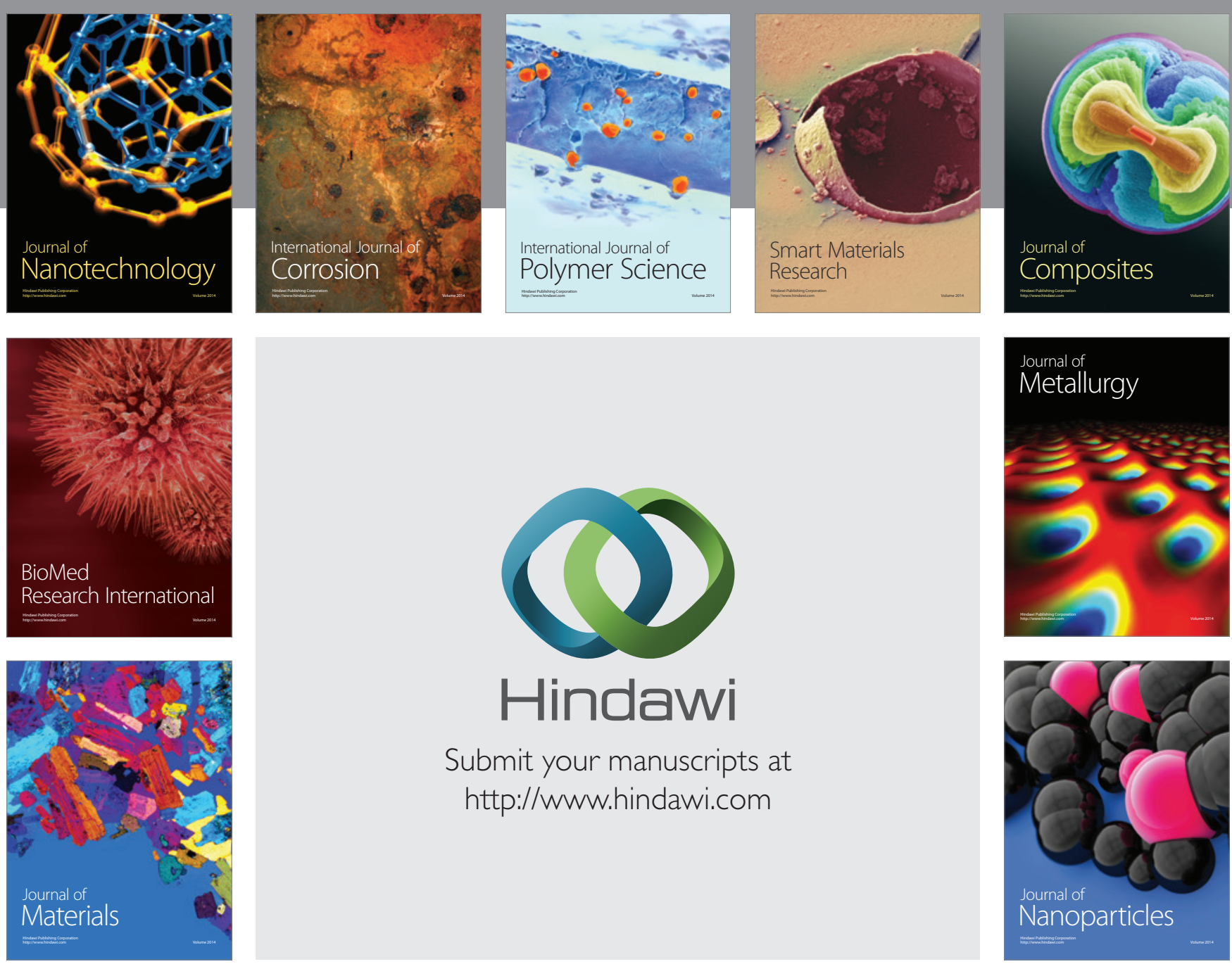

Submit your manuscripts at http://www.hindawi.com
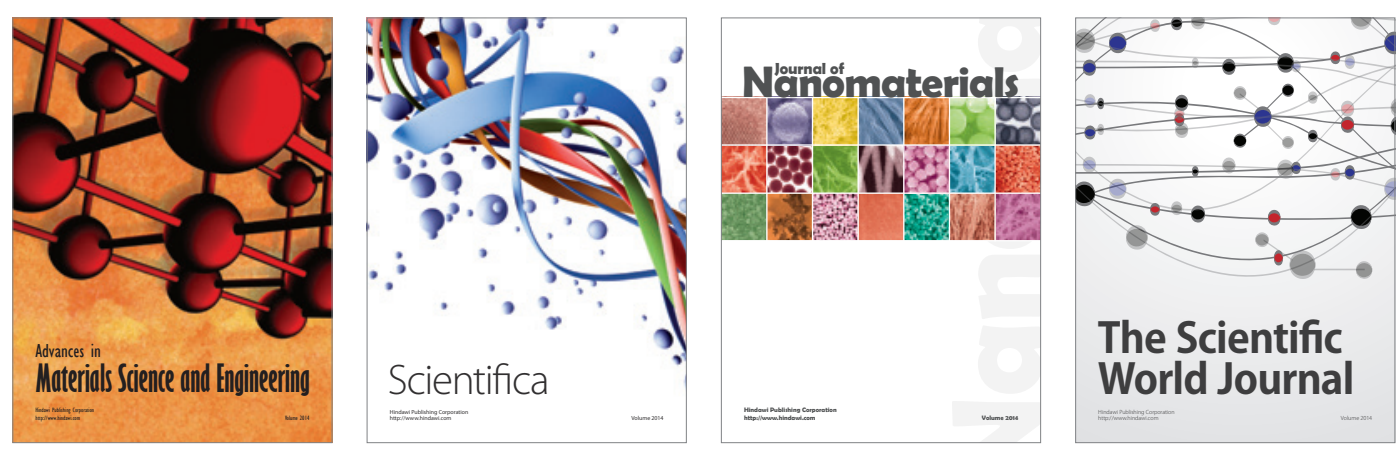

\section{The Scientific World Journal}
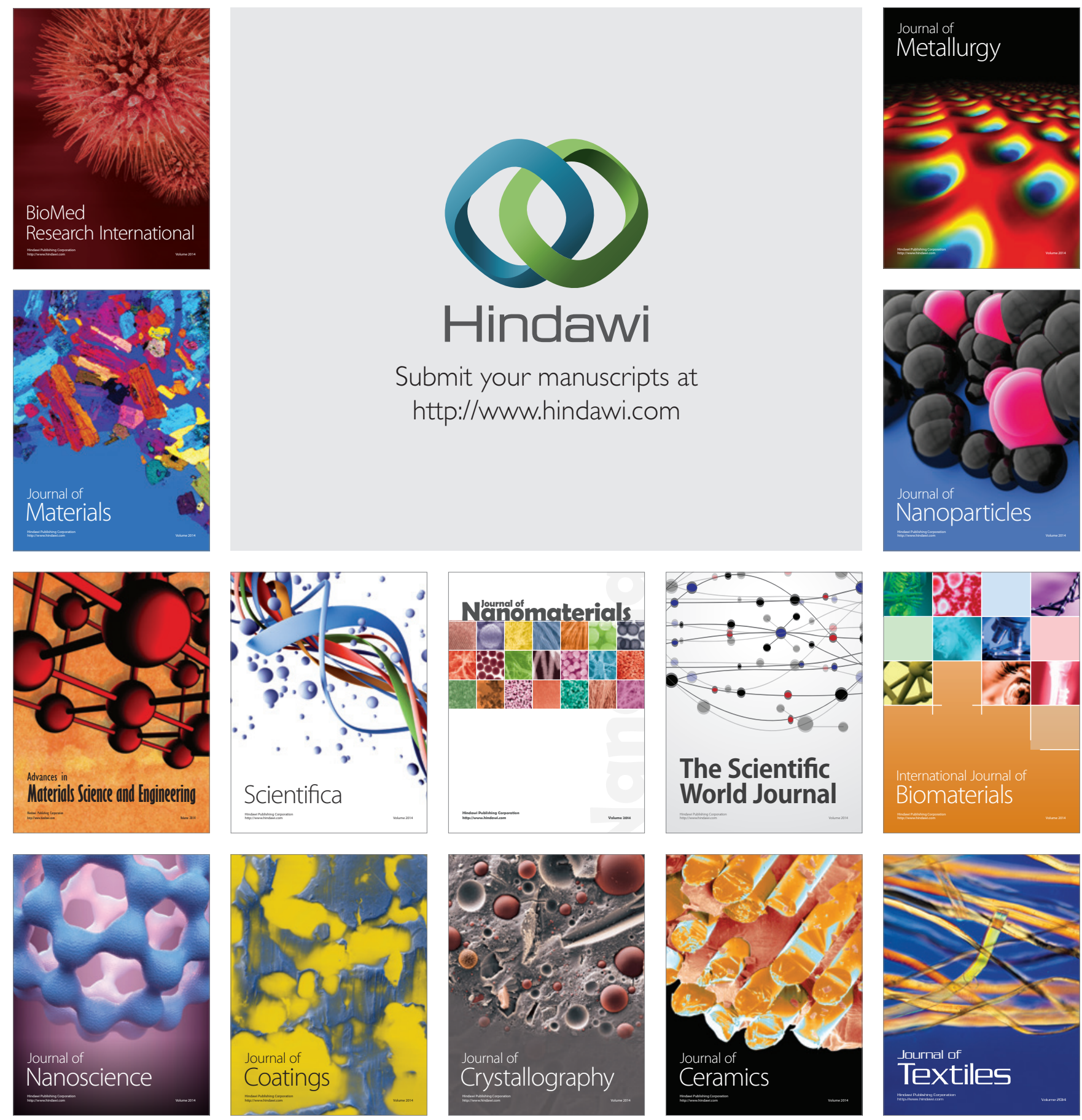